\title{
Drei Kasuistiken eines Granuloma faciale - Stand der aktuellen Therapie
}

\section{Three Cases of Granuloma faciale - Review of Current Treatment Options}

Autoren

Institut
M. Hoefeld-Fegeler, C. Hallermann, H.-J. Schulze

Fachklinik Hornheide, Münster
Bibliografie

DOI http://dx.doi.org/ $10.1055 / \mathrm{s}-0030-1256681$

Online-Publikation: 26. 7. 2011

Akt Dermatol 2011; 37:

363-365 @ Georg Thieme

Verlag KG Stuttgart · New York ISSN 0340-2541

Korrespondenzadresse Dr. Marc Hoefeld-Fegeler Fachklinik Hornheide Dorbaumstraße 300 48157 Münster marc.hoefeld-fegeler@ fachklinik-hornheide.de

\section{Zusammenfassung \\ $\nabla$}

Bei dem Granuloma faciale (GF, synonym: Granuloma faciale eosinophilicum) handelt es sich um eine lokalisierte, chronisch persistierende und therapieresistente, inflammatorische Dermatose

\section{Fall 1}

\section{$\nabla$}

38-jährige, in Deutschland lebende, gebürtige Asiatin. Seit einigen Wochen rasch an Umfang zunehmende und konfluierende Knötchen an der rechten Wange. Urlaubsanamnese im Ausland leer.

Hautbefund ( $\mathbf{A b b} .1 \mathrm{a}$ ): Bis $\mathrm{zu} 2,5 \mathrm{~cm}$ große, bizarr geformte, rosafarbene bis hellbraune Plaque über dem rechten Jochbogen, im unteren Anteil hypopigmentiert und derb infiltriert.

\section{Fall 2}

$\nabla$

46-jährige Patientin. Vor 20 Monaten operative Entfernung eines basaliomverdächtigen Tumors am Nasenrücken, der histologisch die Diagnose eines GF ergab. Seit 11 Monaten gleichartige Knötchen an Nasenwurzel und erneut am Nasenrücken.

Hautbefund ( $\triangle$ Abb. 1 b): Zwei bis zu $1 \mathrm{~cm}$ große, braun- bis lividrote Plaques an der Nasenwurzel links und am Nasenrücken, diaskopisch graubraun.

\section{Fall 3}

$\nabla$

52-jähriger Patient. Seit 8 Wochen ein zunächst lilafarbener Fleck an der rechten Wange mit rascher Größen- und Dickenzunahme. unbekannter Ätiologie mit Hauptmanifestation meist bei Erwachsenen im Gesicht. Anhand von 3 Kasuistiken soll die aktuelle Literatur mit Augenmerk auf gängige Therapieoptionen des GF diskutiert werden.

Hautbefund ( $\bullet$ Abb. 1c): Bis zu $1,2 \mathrm{~cm}$ große, scharf begrenzte, weiche, rot-bräunliche Plaque an der rechten Wange.

\section{Übrige Befunde \\ $\nabla$}

Auch auflichtmikroskopisch in allen 3 Fällen ortsständige Follikelöffnungen erhalten, kein Pigmentnetz und keine radiär ausstrahlenden Teleangiektasien nachweisbar. Regionäre Lymphknoten palpatorisch nicht pathologisch vergrößert. Anamnestisch kein Hinweis auf Diabetes mellitus oder rheumatoide Arthritis.

\section{Histologie \\ $\nabla$}

Unterhalb einer flach papulös über das Hautniveau elevierten Epidermis mit verstrichenen Reteleisten, von dieser durch eine freie Grenzzone getrennt, ein dichtes, histiozytär granulomatöses Infiltrat mit vielen Lymphozyten, einzelnen Plasmazellen und wechselnd vielen eosinophilen Granulozyten bis in die tiefe Dermis reichend und ortsständige Hautadnexe ummantelnd $(\bullet$ Abb. 2a-c). Immunhistologisch exprimieren die histiozytären Zellen CD68, die lymphoiden Zellen CD3 und vereinzelt CD30-Antigen. 


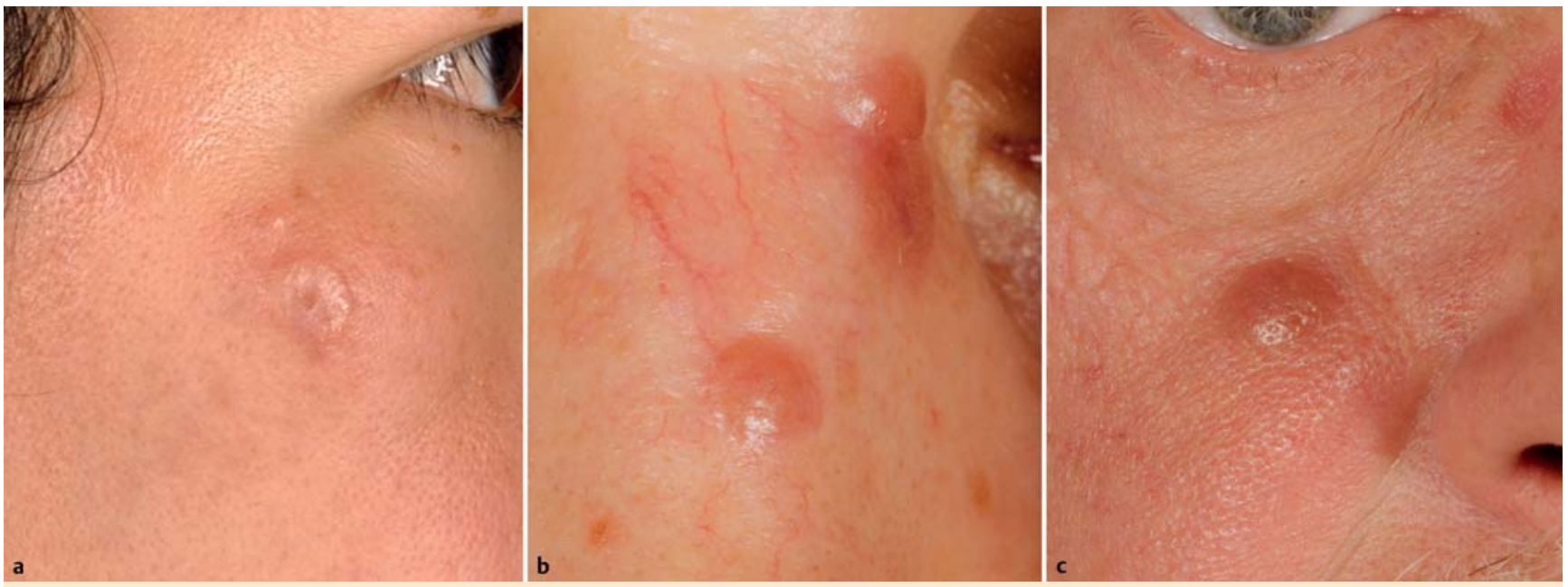

Abb. 1 a Fall 1; b Fall 2; c Fall 3.
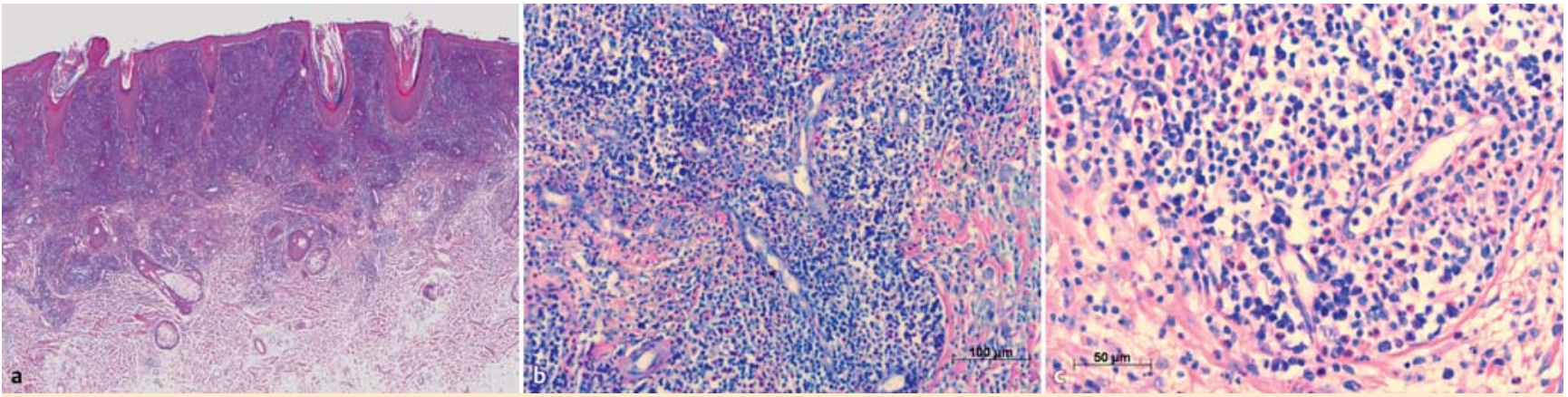

Abb. 2 a HE, $\times$ 4; b HE, $\times 20$; c Giemsa, $\times 400$.

\section{Diskussion}

$\nabla$

Das Krankheitsbild des Granuloma faciale, erstmals von Wigley im Jahr 1945 beschrieben, wurde 1950 von Lever und Leeper als distinkte klinische und histologische Entität herausgestellt, der Name wurde im selben Jahr von Pinkus u. Mitarb. vorgeschlagen. Das GF ist eine seltene, gutartige, chronisch verlaufende Hautkrankheit, die meist bei Weißhäutigen gesehen wird. Einzelfallbeobachtungen bei Japanern und Schwarzhäutigen liegen vor. Es betrifft Männer häufiger als Frauen und tritt meist im mittleren Lebensalter auf (Median: 45 Jahre). Typisch sind solitäre oder multiple, kutane Knoten im Gesicht, bei gelegentlich extrafazialer Manifestation meist in lichtexponierten Hautarealen, insbesondere behaarter Kopfhaut, Rumpf und oberen Extremitäten. Berichte von GF-artigen Effloreszenzen an der Mundschleimhaut sind selten [1-3]. Das GF verläuft gewöhnlich asymptomatisch, selten werden Druckschmerz, Juckreiz oder Brennen berichtet. Der Durchmesser der Effloreszenzen variiert von wenigen Millimetern bis zu mehreren Zentimetern, anfangs sieht man ein livid-rotes Erythem, später braun- bis lividrote, umschriebene Plaques und Knoten mit glatter Oberfläche und erweiterten Follikelmündungen, wodurch der „orangenschalenartige“ Oberflächenaspekt entsteht. Bei Sonnenexposition werden die Läsionen rasch dunkelbraun. Die Effloreszenzen imitieren viele Dermatosen, insbesondere kutane Sarkoidose, (subakut-kutaner) Lupus erythematodes, kutanes Pseudolymphom oder Lymphom und fixes Arzneimittelexanthem, als weitere klinische Differenzialdiagnosen sind vor allem zu nennen: Akute febrile neutrophile Dermatose, Alopecia mucinosa, noduläre lokalisierte kutane
Amyloidose, angiolymphoide Hyperplasie mit Eosinophilie, kutane Leishmaniose, kutanes Lymphozytom, lymphozytäre Infiltration der Haut (Jessner) oder Tuberculosis cutis luposa.

Ursächlich wird eine lokalisierte, chronische ImmunkomplexVaskulitis mit IgG-Ablagerungen in den Gefäßwänden diskutiert. Die Produktion von Interleukin 5 durch die klonale T-Zell-Population scheint verantwortlich für die Einwanderung der Eosinophilen in das dermale Infiltrat. Ätiopathologisch könnte auch die UV-Exposition eine Rolle spielen, weil sonnenexponierte Haut viel häufiger betroffen ist als nicht UV-exponierte Hautareale. Histologisch sieht man im Frühstadium neutrophile Granulozyten und „Kernstaub“ wechselnd dicht nodulär bis diffus verteilt interstitiell in der oberen Hälfte der retikulären Dermis, zudem Lymphozyten und wechselnd viele Eosinophile perivaskulär und Fibrin in der Gefäßwand [4]. Im voll ausgeprägten Stadium finden sich zusätzlich Plasmazellen und Histiozyten im dermalen Infiltrat, im Spätstadium zusätzlich vermehrt Fibroblasten und kabelartige Bündel kollagener Fasern in storiformem Muster. Diagnostisch wegweisend ist die Aussparung der papillären und periadnexiellen Dermis in allen Krankheitsphasen des GF.

Nach histologischer Diagnosesicherung in einer klinisch repräsentativen Hautbiopsie ist bei Behandlungswunsch aus einer Vielzahl von Therapieverfahren zu wählen. Als operative Verfahren steht neben der Exzision und Dermabrasion die Ablation mittels $\mathrm{CO}_{2}$ - oder Erbium-Yag-Laser, Elektrokauter oder Radiotom zur Verfügung, ferner die Behandlung mit dem Argon- oder Farbstoff-Laser, zudem die Kryochirurgie. Angesichts seines geringen Narbenrisikos ist der gepulste Farbstoff-Laser $(585 \mathrm{~nm})$ zu bevorzugen [5]. 
In den o.g. Fällen behandelten wir jeweils einmal mit einem kortisonhaltigen Externum, mittels intraläsionaler Einspritzung von Triamcinolonacetonid-Kristallsuspension sowie Exzision in Lokalanästhesie. Letztere Therapie stellt aufgrund der Narbenbildung nicht die erste Wahl da, wurde jedoch aufgrund eines sehr begrenzten Befundes und des hohen Leidensdruckes des Patienten mit gutem Erfolg durchgeführt.

Wegen der häufig frustranen operativen Therapieversuche werden viele verschiedene medikamentöse Verfahren bei GF empirisch und individuell eingesetzt. Angesichts der Remission ohne Narbenbildung auch nach wiederholter Behandlung mit dem gepulsten Farbstoff-Laser ist dieser einer medikamentösen Langzeittherapie vorzuziehen. Medikamentös unterschiedlich gut wirksam sind topische Kortikosteroide oder eine Kryotherapie [6,7], besser eine „first-line“ intraläsionale Glukokortikosteroidinjektion mit Triamcinolonacetonid-Kristallsuspension, ferner intraläsionale Goldinjektionen, Einnahme von Antimalariamitteln, Calciferol, Isoniazid, Paraaminobenzoesäure und Bismuth, zudem lokale PUVA-Therapie, Röntgenweichstrahltherapie und topische Anwendung von Tacrolimus [8]. Letzteres wirkt entzündungshemmend durch Suppression der Freisetzung von Zytokinen in T-Zellen und Hemmung der im Frühstadium der T-ZellAktivierung involvierten Gen-Transkription von IL-3, IL-4, IL-5, GM-CSF und TNF-alpha. Es hemmt ferner die Freisetzung von entzündlichen Mediatoren in kutanen Mastzellen und Basophilen sowie die Expression von FCeRI auf Langerhanszellen.

Die meisten Berichte über eine wirksame orale Behandlung liegen für die antimikrobiellen Substanzen Dapsone und Clofazimine vor. Dapsone (DADPS) weist neben potenten antiinflammatorischen Eigenschaften bei vielen entzündlichen Hauterkrankungen vor allem bakterizide und bakteriostatische Wirkung gegen Mykobakterien auf, vergleichbar mit der von Sulfonamiden, und verhindert als kompetitiver Antagonist von PABA die Bildung von Folsäure und damit das Bakterienwachstum. Zur Prävention einer Methämoglobinämie ist vor Therapiebeginn die serologische Bestimmung der Glukose-6-Phosphat-DehydrogenaseAktivität zu empfehlen. Clofazimine (Lamprene) fördert in Neutrophilen und Monozyten die Phagozytose und Freisetzung lysosomaler Enzyme und hemmt die Neutrophilenmotilität und Lymphozytentransformation.
Zusammenfassend sollte der Patient aufgrund des chronisch persistierenden und häufig therapieresistenten Verlaufs mit zumeist atrophisch narbiger Abheilung frühzeitig über das Krankheitsbild und das hohe Rezidivrisiko bei alleiniger ablativer Therapie informiert werden. Aufgrund ihrer guten Ansprechraten sollten vor allem lokale, nicht-invasive Therapieverfahren und hier vor allem die Behandlung mit dem gepulsten FarbstoffLaser unter Nutzen-Risiko-Abwägung der gutartigen Dermatose empfohlen werden.

\section{Abstract}

\section{Three Cases of Granuloma faciale - Review of Current Treatment Options \\ $\nabla$}

Granuloma faciale (GF) is a benign localized chronic persistent inflammatory dermatosis of unknown aetiology usually appearing in the face. On the basis of three illustrative cases we briefly review the current literature with attention to available treatment options.

\section{Literatur}

1 Nasiri S, Rahimi H, Farnaghi A, Asadi-Kani Z. Granuloma faciale with disseminated extra facial lesions. Dermatol Online J 2010 Jun 15; 16 (6): 5

2 Rosiello L, Palla M, Aiello FS et al. Granuloma faciale with extrafacial lesions. Skinmed 2007; 6: 150-151

3 Ortonne N, Wechsler J, Bagot $M$ et al. Granuloma faciale: a clinicopathologic study of 66 patients. J Am Acad Dermatol 2005; 53: $1002-1009$

4 Khaled A, Jones $M$, Zermani $R$ et al. Granuloma faciale. Pathologica 2007; 99: 306-308

5 Fikrle T, Pizinger K. Granuloma faciale treated with 595-nm Pulsed Dye Laser. Dermatol Surg 2011; 37: 102 - 104

6 Rosiello L, Palla M, Aiello FS et al. Granuloma faciale with extrafacial lesions. Skinmed 2007; 6: 150-151

7 Panagiotopulos A, Anyfantakis V, Rallis E et al. Assessment of the efficacy of cryosurgery in the treatment of granuloma faciale. $\mathrm{Br} \mathrm{J}$ Dermatol 2006; 154: $357-360$

8 Cecchi R, Pavesi M, Bartoli L, Brunetti L. Topical tacrolimus in the treatment of granuloma faciale. Int J Dermatol 2010; 49: 1463 - 1465 